\title{
Reliable Provisioning with Degraded Service Using Multipath Routing from Multiple Data Centers in Optical Metro Networks
}

This paper was downloaded from TechRxiv (https://www.techrxiv.org).

LICENSE

CC BY 4.0

SUBMISSION DATE / POSTED DATE

01-03-2022 / 03-03-2022

\section{CITATION}

Le, Giap (2022): Reliable Provisioning with Degraded Service Using Multipath Routing from Multiple Data Centers in Optical Metro Networks. TechRxiv. Preprint. https://doi.org/10.36227/techrxiv.19255001.v1

DOI

10.36227/techrxiv.19255001.v1 


\begin{abstract}
With the adoption of edge computing, several data centers are available within the footprint of an optical metro network, and contents are replicated in multiple locations. Such a wide content replication offers a unique opportunity to provide better services to users, especially for content-based services, e.g., video delivery. Thus, a service-provisioning scheme can embrace this opportunity to optimize network resource utilization, improve reliability, and achieve lower latency. In this study, we propose a reliable service-provisioning scheme that selects the optimal subset of data centers hosting the desired content and inversely multiplexes a content request over multiple linkdisjoint paths. We formulate an integer linear program and develop heuristics for the problem, and use them to solve various complex and realistic network instances. Numerical data show that, compared to conventional service-provisioning schemes such as multipath routing from a single data center or dedicatedpath protection, our proposed scheme efficiently utilizes network resources, improves reliability, and reduces latency; hence, it is suitable for the above-mentioned services.
\end{abstract}

Index Terms-Optical Metro Networks, Reliable Service Provisioning, Degraded Service, Content Connectivity, Multipath Routing, Manycast.

\section{INTRODUCTION}

$\mathbf{O}$ TICAL metro networks are attracting significant investments to evolve from a rigid ring-based aggregation infrastructure to a composite network-and-computing ecosystem where new applications and services can be implemented and supported [1]. In particular, with the adoption of edge computing, several data centers (DCs) are now available within the footprint of an optical metro network. Typically, micro DCs are available in metro-access nodes, medium-size DCs are available in metro-core nodes, while hyper-scale DCs are available in core network nodes, and they communicate with the metro network via metro-core backbone nodes as gateways. Hence, with edge computing, contents (e.g., media files, applications, web services, and documents) are now widely replicated in multiple DCs closer to users to offload core network traffic and to lower latency [2], [3].

Such a wide replication of contents in multiple locations offers a unique opportunity to provide better services to users, especially for content-based services. Among such services, video delivery is playing the main role, as, by 2022 , it will be $79 \%$ of the world's mobile data traffic and $82 \%$ of all consumer Internet traffic [4]. Other content-based services, such as augmented reality (AR) and virtual reality (VR),

Corresponding author: Giap Le, dgle@ucdavis.edu are emerging, which allow users to interact intuitively with the environment through six degrees of freedom [5]. These services require high bandwidth, low latency, reliable connectivity, and are classified as mobile broadband reliable low-latency communication (MBRLLC) in the vision of $6 \mathrm{G}$ communications [6]. While some of these services require full protection, others can continue to operate with reduced, i.e., degraded, quality in case of failures and can be served with partial protection (e.g., a video stream can switch to a lower resolution depending on available bandwidth).

High-capacity optical metro networks are exposed to many threats such as malicious attacks, equipment failures, human errors (e.g., misconfigurations), and natural and human-made large-scale disasters (e.g., earthquakes, hurricanes, and terrorist attacks). To ensure reliability, protection and restoration schemes are traditionally used. In a protection scheme, extra network resources are reserved when a connection is provisioned. Conventionally, a pair of paths is provided to a connection: one is used to carry traffic during normal operation, referred to as primary path; and the other path, referred to as backup path, is reserved and will be activated after a failure occurs on the primary path. In a restoration scheme, no extra resources are reserved for the backup path, and the network must react to find an alternative path after a failure occurs on the primary path [7]. Since failures are hard to predict and statistically rare, providing protection in a dynamic network environment, especially full protection against multiple failures, would require massive and economicallyunsustainable bandwidth overprovisioning. On the other hand, a restoration scheme would require a longer recovery time and provide no guarantee to restore a disrupted path.

This study concentrates on multipath routing, a flexible and resource-efficient protection scheme in which a service request is provisioned over multiple paths by routing part of the requested bandwidth on each path [8], [9]. With respect to the baseline multipath routing, we consider the opportunity to route different paths towards different destinations, representing different metro DCs hosting the required content. To illustrate our proposed service-provisioning scheme, in Fig. 1, we consider a dynamic network environment where, at time $t$, a user at node 1 is requesting for a content replicated in multiple DCs at nodes 4, 5, 6, and 7. In addition, the user requires bandwidth $b$ during normal operation and can tolerate degraded service (i.e., degraded bandwidth) $0.6 b$ in case of a single-link failure. Note that, even though multiple-link/node 


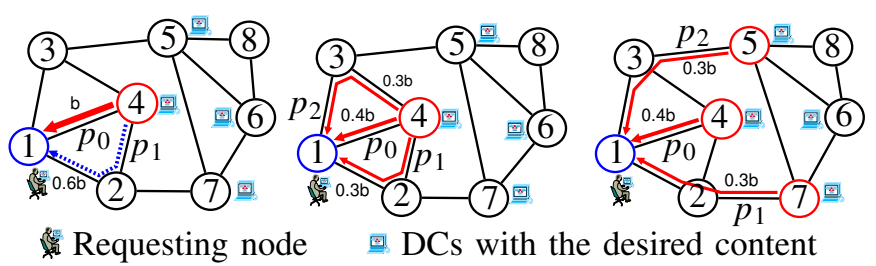
a. DPP
b. MPSD
c. MPMD

failures can occur, a single-link failure is still the dominant failure scenario in an optical network [10]-[13]. Also, due to the asymmetric traffic characterizing content retrieval, we only consider downstream traffic (i.e., from DCs to the requesting node). Conventionally, a dedicated-path protection (DPP) scheme selects the optimal DC (e.g., the DC at node 4 which is closest to the requesting node) and reserves a pair of primary and backup paths (e.g., $p_{0}$ and $p_{1}$ ) for the request [14]. As shown in Fig. 1.a, the bandwidths on the primary and backup paths are $b$ and $0.6 b$, respectively. In case of a failure occurring on the primary path, the requested degraded service is still guaranteed after the backup path is activated. In total, DPP requires bandwidth $1.6 b$ over the primary and backup paths and occupies network bandwidth $2.2 b$. Here, we define the network bandwidth as the sum of the bandwidth on each path weighted by the number of hops (i.e., $2.2 b=b+2 * 0.6 b$ ).

Fig. 1.b shows a different protection scheme using multipath routing from a single DC (MPSD) [8], [9], [15]-[17]. In this scenario, data from the closest DC at node 4 to the requesting node are simultaneously transmitted on three linkdisjoint paths $p_{0}, p_{1}$, and $p_{2}$ with bandwidths of $0.4 b, 0.3 b$, and $0.3 b$, respectively. In case a failure occurs on a path, the requested degraded service is still fulfilled since survivable bandwidth remains at least $0.6 b$. In total, MPSD requires bandwidth $b$ over three paths and occupies network bandwidth 1.6b. In Figs. 1.a and 1.b, DPP and MPSD share a major shortcoming as they do not provide protection against failures in the source DC. For instance, in case of a failure occurring in the DC at node 4, provisioned services are disrupted.

In Fig. 1.c, we describe a service-provisioning scheme where the user at node 1 is simultaneously served by three different DCs at nodes 4,5 , and 7 on three link-disjoint paths $p_{0}, p_{1}$, and $p_{2}$ with bandwidths of $0.4 b, 0.3 b$, and $0.3 b$, respectively. Hereafter, we refer to this service-provisioning scheme as multipath routing from multiple DCs (MPMD). In case a path is disrupted, MPMD ensures that survivable bandwidth remains at least $0.6 b$; hence, the required degraded service is still guaranteed. Compared to DPP and MPSD, MPMD also provides protection against failures in DCs (i.e., survivable bandwidth remains at least $0.6 b$ if a failure occurs in one of the serving DCs, e.g., at node 4). In total, MPMD requires bandwidth $b$ over three paths and occupies network bandwidth $1.6 b$. For each content request, MPMD must: 1) select the optimal subset of DCs hosting the desired content, 2) find link-disjoint paths from each selected DC to the requesting node, and 3) allocate bandwidth to each path such that the total requested bandwidth during normal operation and degraded

service in case a path is disrupted are fulfilled. In literature, this service-provisioning scheme is often referred as an inverse manycast scheme to indicate that source nodes (i.e., optimal subset of DCs) must be selected from a larger candidate set [13], [18]. Compared to an inverse multicast scheme where source nodes are specified ahead of time, an inverse manycast scheme has greater flexibility in choosing the source nodes from which data (e.g., contents) are retrieved.

In this study, for the first time and to the best of our knowledge, we propose MPMD as a reliable service-provisioning scheme to fulfill content requests (e.g., video-on-demand, VR, or $\mathrm{AR}$ requests) in a dynamic network environment while guaranteeing strict requirements on bandwidth, and improving reliability and latency. This proposed service-provisioning scheme enjoys the benefits of multipath routing, provides protection against network (e.g., link) and content source (e.g., DC) failures, and uses minimal additional network resources due to the nature of multipath routing. We formulate the MPMD problem as an integer linear program (ILP), develop two scalable heuristics, and use them to solve various complex network instances. Numerical data show that, compared to conventional service-provisioning schemes such as DPP and MPSD, MPMD efficiently utilizes network resources, provides higher reliability, reduces latency; hence, it is highly suitable for the emerging content services.

The rest of this study is organized as follows. In Section II, we review related works. In Section III, we formulate the MPMD problem as an ILP. In Section IV, we develop heuristics for the MPMD problem. In Section V, we perform numerical validation in various scenarios and compare the performance of MPMD to those of reference protection strategies (e.g., MPSD, DPP). We conclude this study in Section VI.

\section{RELATED WORKS}

Some studies have been conducted on reliable service provisioning using multipath routing. In [8], [9], [15], the authors developed algorithms for multipath routing in a static scenario in which, for each traffic demand, the source and destination nodes are specified ahead of time. Due to the nature of multipath routing, the algorithms ensure a certain level of degraded service in case of failures on one or several paths. In [16], the authors extended their work in [15] to a dynamic scenario where survivable bandwidth is guaranteed in case a path is disrupted. In [18], the authors studied the problem of routing and wavelength assignment for static manycast demands in wavelength-division multiplexing (WDM) networks. They proposed a solution for upstream traffic in which the requesting node sends data to several nodes in a larger set of candidate nodes using multipath routing and manycast.

The authors in [19] leveraged the concept of content connectivity in DC networks and developed algorithms to place contents at optimal DCs for a static scenario such that $K$ node/link-disjoint paths are guaranteed from the requesting node to the DCs hosting the desired content. In [20], the authors proposed a reliable service-provisioning scheme using multipath routing and inverse manycast. However, the authors developed the solution for a static scenario where the path 
between each pair of nodes is pre-computed. This assumption makes the solution less practical for content-retrieval applications where requests (e.g., video on demand) arrive, hold, and depart dynamically, and the network topology changes over time. In this study, our focus is on the dynamic problem for content requests leveraging multipath routing and inverse manycast while ensuring strict requirements on bandwidth, and improving reliability and latency.

\section{Reliable Provisioning With Degraded Service USING MPMD}

In this section, we formally state the MPMD problem and formulate it as an ILP (MPMD-ILP).

\section{A. Problem Statement}

In this study, each dynamic content request, $\theta$, is characterized by a tuple $\theta=(t, n, c, b, m)$ where $t$ is arrival time (s), $n$ is the requesting node, $c$ is the desired content, $b$ is requested bandwidth (Mbps), and $m$ is the ratio of survivable bandwidth to requested bandwidth in case a path is disrupted. In other words, if a path is disrupted, survivable bandwidth must remain at least $m * b$. We consider a graph $G_{t}\left(V_{t}, E_{t}\right)$ to represent a network where $V_{t}$ is the set of nodes with available computing capacity and $E_{t}$ is the set of links with available bandwidth at request arrival time. Since content requests come, hold, and depart, the sets $V_{t}$ and $E_{t}$ can vary over time. The desired content, $c$, has size $h(\mathrm{~GB})$, and is replicated in multiple DCs denoted by the set of nodes $D, D \subset V_{t},|D| \geq 2$. Without loss of generality, the requesting node does not host the desired content, i.e., $n \notin D$. Also, we assume that each selected DC can support only one path. To guarantee degraded service (i.e., bandwidth $m^{*} b$ ) in case a path is disrupted, total offered bandwidth over all paths, $b^{\prime}$ (Mbps), can be larger than requested bandwidth (i.e., $b^{\prime} \geq b$ ). Moreover, if a request is offered bandwidth $b^{\prime}$, it departs at $t^{\prime}=t+8000 * h / b^{\prime}$ (s), where $8000 * h / b^{\prime}$ is the holding time (s) of the request (i.e., $h$ (GB) and $b^{\prime}$ (Mbps)). Note that we consider large contents, so a content's transmission delay is the major contributor to its total delay (and its propagation delay through the network is relatively negligible).

The MPMD problem can be formally stated as follows. Given the content request $\theta=(t, n, c, b, m)$, the network graph at request arrival $G_{t}\left(V_{t}, E_{t}\right)$, and availability of the desired content characterized by its size $h$ and set of hosting DCs $D$, find: 1) optimal subset of DCs hosting the desired content (for convenience, we use $D_{0}$ to denote this optimal subset, $\left.D_{0} \subseteq D\right)$, 2) link-disjoint paths from each DC in $D_{0}$ to requesting node $n$, and 3) bandwidth on each path such that total requested bandwidth $b$ during normal operation and degraded service $m * b$ in case a path is disrupted are fulfilled.

Noting that multiple objectives cannot be optimized simultaneously, a weighted objective function needs to be defined. The objective is to minimize total bandwidth over all paths, total network bandwidth (weighted sum), total propagation delay of the paths from each selected DC to the requesting node, and total risk of the physical links on which the paths are mapped. Here, each physical link is characterized by a normalized risk factor based on its physical location. We elaborate on our risk model for a physical network in the next section.

The optimal solution is subject to constraints on the available capacity of each physical link, maximum differential delay between paths (i.e., differential delay constraint (DDC) which is maximum difference of propagation and processing delay between paths that can be compensated at the receiver [17]), and survivability of paths.

\section{B. Mathematical Formulation}

Inputs:

- $G_{t}\left(V_{t}, E_{t}\right)$ : network graph at request arrival time.

- $\theta=(t, n, c, b, m)$ : content request.

- $h$ : size of the desired content (GB).

- $D$ : DCs hosting the desired content, $D \subset V_{t},|D| \geq 2$.

- $\Lambda_{t}$ : hash table representing capacity of physical links where each key-value pair $(i, j): \lambda_{t}^{i, j}, \forall(i, j) \in E_{t}$, is link $(i, j)$ 's available capacity at request arrival time (Mbps).

- $\Xi$ : hash table representing propagation delay of physical links with each key-value pair $(i, j): \xi^{i, j}, \forall(i, j) \in E_{t}$, being propagation delay of link $(i, j)(\mu \mathrm{s})$.

- $\Phi$ : hash table representing risk factors of physical links with each key-value pair $(i, j): \varphi^{i, j}, \forall(i, j) \in E_{t}$, being normalized risk factor of link $(i, j)$.

- $\Omega$ : maximum differential delay between paths $(\mu \mathrm{s})$.

\section{Variables:}

- $w_{d, n}$ : a binary variable, and $w_{d, n}=1$ if requesting node $n$ uses DC $d, d \in D$; and 0 otherwise.

- $x_{d, n}$ : an integer variable denoting the bandwidth reserved on the path from DC $d, d \in D$, to requesting node $n$.

- $y_{d, n}^{i, j}$ : an integer variable denoting the mapping of the path from DC $d, d \in D$, to requesting node $n$ on physical links $(i, j)$. Here, $y_{d, n}^{i, j}=x_{d, n}, x_{d, n}>0$ if the path from DC $d$ to requesting node $n$ is mapped on physical link $(i, j)$; and 0 otherwise.

- $z_{d, n}^{i, j}$ : a binary variable, and $z_{d, n}^{i, j}=1$ if the path from DC $d, d \in D$, to requesting node $n$ is mapped on link $(i, j)$; and 0 otherwise.

\section{Objective function:}

$$
\begin{gathered}
\min _{\forall n}\left(\alpha * \sum_{d \in D} x_{d, n}+\beta * \sum_{\substack{d \in D,(i, j) \in E_{t}}} y_{d, n}^{i, j}\right. \\
\left.+\gamma * \sum_{\substack{d \in D,(i, j) \in E_{t}}} \xi^{i, j} z_{d, n}^{i, j}+\delta * \sum_{\substack{d \in D,(i, j) \in E_{t}}} \varphi^{i, j} z_{d, n}^{i, j}\right)
\end{gathered}
$$

Subject to:

$$
\sum_{d \in D} w_{d, n} \geq 2, \forall n
$$




$$
\begin{aligned}
& \sum_{d \in D} x_{d, n} \geq b, \quad \forall n \\
& w_{d, n} \geq x_{d, n} / \Psi, \quad \forall d \in D, \forall n . \\
& \sum_{d \in D} y_{d, n}^{i, j} \leq \lambda_{t}^{i, j}, \quad \forall(i, j) \in E_{t}, \forall n .
\end{aligned}
$$

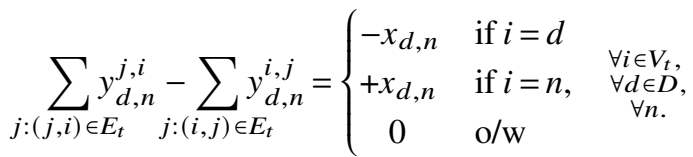

$$
\begin{aligned}
& z_{d, n}^{i, j} \geq y_{d, n}^{i, j} / \Psi, \quad \forall d \in D, \forall(i, j) \in E_{t}, \forall n . \\
& x_{d, n}-\sum_{d \in D} x_{d, n} \leq-m^{*} b, \quad \forall d \in D, \forall n . \\
& \sum_{d \in D} z_{d, n}^{i, j} \leq 1, \quad \forall(i, j) \in E_{t}, \forall n . \\
& \left|\sum_{(i, j) \in E_{t}} \xi^{i, j} z_{d_{1}, n}^{i, j}-\sum_{(i, j) \in E_{t}} \xi^{i, j} z_{d_{2}, n}^{i, j}\right| \leq \Omega, \\
& \forall d_{1}, d_{2} \in D: d_{1} \neq d_{2}, \forall n .
\end{aligned}
$$

In objective function (1), we introduce the scalars $\alpha, \beta, \gamma$, and $\delta$ to control the weight of each term. The first summation minimizes total bandwidth over all paths while the second summation minimizes total network bandwidth. The first and second terms of the objective function minimize total network resources reserved for the request; since $\alpha>\beta \gg \gamma>\delta$ (e.g., $\alpha=50000, \beta=40000, \gamma=1000$, and $\delta=500$ ), they are also the leading terms. The third summation minimizes total propagation delay of the paths from each DC in $D_{0}$ to the requesting node. As a result, DCs close to the requesting node are generally selected. The fourth summation minimizes total normalized risk factor of physical links on which the paths from each DC in $D_{0}$ to the requesting node are mapped. Hence, the fourth term guarantees that the paths from each DC in $D_{0}$ to the requesting node are mapped on physical links with lower risk.

Constraint (2) ensures that each request is simultaneously served by at least two DCs (i.e., multiple DCs). Constraint (3) guarantees that the total bandwidth over all paths for each request is at least $b$ during normal operation. Constraint (4) sets the binary variable $w_{d, n}=1$ if the requesting node $n$ uses DC $d$ (i.e., $x_{d, n}>0$ ) where $\Psi$ is a large positive integer, e.g., 10000. Constraint (5) requires that the mapping of the request on the physical network does not exceed the capacity of each physical link at request arrival time. Constraint (6) enforces flow conservation in which, for each path, traffic originates from DC $d$ and ends at requesting node $n$. At a transit node, input traffic is equal to output traffic. Constraint (7) computes a binarization of the integer variable $y_{d, n}^{i, j}$ and assigns it to $z_{d, n}^{i, j}$. Constraint (8) guarantees that the desired degraded service is satisfied (i.e., survivable bandwidth must remain at least $m * b$ in case a path is disrupted). Constraint (9) restricts the

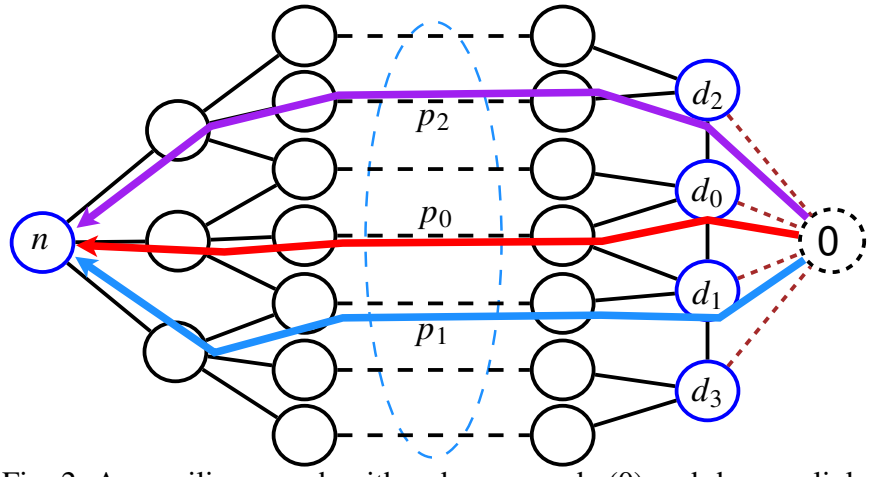

Fig. 2: An auxiliary graph with a dummy node (0) and dummy links (dotted lines from node 0 to DCs hosting the desired content).

mapping of the request on the physical network such that a single physical link cannot be shared by two or more paths. In other words, traffic from the DCs in $D_{0}$ to the requesting node is carried on link-disjoint paths. Therefore, constraints (8) and (9) strictly enforce survivable bandwidth to be at least $m * b$ in case a path is disrupted. Constraint (10) ensures that the differential delay of two distinct paths fulfills DDC.

In our MPMD-ILP, the numbers of variables and constraints for each request (i.e., $|n|=1$ ) are upper bounded by $2 *|D| *\left(1+\left|E_{t}\right|\right)$ and $2 *\left(1+|D|+\left|E_{t}\right|\right)+|D| *\left(\left|V_{t}\right|+\left|E_{t}\right|\right)+\left(\begin{array}{c}|D| \\ 2\end{array}\right)$, respectively. Here, we use $|\cdot|$ to denote the cardinality of a set and $($.$) is the combination without repetition. Since \left(\begin{array}{c}|D| \\ 2\end{array}\right)$ can be reduced to $|D| *(|D|-1) / 2$, the number of variables increases linearly with number of hosting DCs and number of physical links, the number of constraints increases linearly with number of physical nodes and number of physical links, while it increases quadratically with number of hosting DCs.

\section{Heuristics For Reliable Provisioning with DEgRADED SERVICE USING MPMD}

Since the MPMD-ILP presented in Section III is intractable for large network instances, it is impractical in a dynamic network environment where a fast solution is more desirable. In this section, we propose various heuristic algorithms for reliable provisioning with degraded service using MPMD. Before that, below, we introduce the auxiliary graph used in the following heuristics.

\section{A. Auxiliary Graph}

To find link-disjoint paths from the DCs hosting the desired content to the requesting node, we introduce an auxiliary graph by leveraging a dummy node and several dummy links. To ensure that the final output is not affected by the addition of the dummy node and links, we assume that the dummy node has unlimited computing capacity and the dummy links have unlimited bandwidth and zero propagation delay.

As shown in Fig. 2, the dummy node (i.e., node 0) is connected to each DC hosting the desired content using the dummy links (denoted by dotted lines). The solid and dashed lines represent the network graph at request arrival time (i.e., $\left.G_{t}\left(V_{t}, E_{t}\right)\right)$ in which a user at node $n$ is requesting for a content replicated in DCs $d_{0}, d_{1}, d_{2}$, and $d_{3}$ (i.e., 
$\left.D=\left\{d_{0}, d_{1}, d_{2}, d_{3}\right\}\right)$. Here, we use the dashed lines to abstract the real network with more nodes and links. Henceforth, we use $G_{t}^{d}\left(V_{t}^{d}, E_{t}^{d}\right)$ to denote the auxiliary network graph which includes $G_{t}\left(V_{t}, E_{t}\right)$, the dummy node, and dummy links.

One can observe that, to find the link-disjoint paths from the DCs hosting the desired content to the requesting node, we can find the link-disjoint paths from the dummy node to the requesting node on $G_{t}^{d}\left(V_{t}^{d}, E_{t}^{d}\right)$. Once the link-disjoint paths are found, the MPMD problem in the previous section can be restated as follows. Given the link-disjoint paths from the DCs hosting the desired content to the requesting node, allocate bandwidth to each path such that, for each content request, total requested bandwidth $b$ during normal operation and degraded service $m * b$ in case a path is disrupted are fulfilled. The amount of bandwidth allocated to each path must not exceed the available/bottleneck bandwidth of the path, and differential delay of two distinct paths must satisfy DDC. In the specific scenario in Fig. 2, the numbers of linkdisjoint paths (i.e., $K$ ), also the number of serving DCs, (i.e., $K=\left|D_{0}\right|=3$ ), is fewer than the number of DCs hosting the desired content (i.e., $|D|=4$ ). In this scenario, the algorithm selects the DCs in ascending order of their distances to the requesting node.

\section{B. Equal-Bandwidth, Maximum-K MPMD (K-MPMD)}

In this subsection, we design an algorithm that finds the maximum number of link-disjoint paths from the dummy node to the requesting node, and equally allocates bandwidth to each path such that the total requested bandwidth and degraded service are fulfilled. Hereafter, we refer to this serviceprovisioning scheme as the equal-bandwidth, maximum-K MPMD (K-MPMD).

In Algorithm 1 , as inputs, $\theta=(t, n, c, b, m), G_{t}\left(V_{t}, E_{t}\right)$, $h, D, \Lambda_{t}, \Xi$, and $\Omega$ denote the tuple representing a content request, network graph at request arrival time, desired content size (GB), set of DCs hosting the desired content, hash table representing the available capacity of each link in $E_{t}$ at request arrival time, hash table representing the propagation delay of each link in $E_{t}$, and maximum differential delay between paths $(\mu \mathrm{s})$, respectively. As outputs, Algorithm 1 finds $K$ as the number of link-disjoint paths for the content request, $D_{0}$ as the set of optimal DCs to serve the request $\left(D_{0} \subseteq D,\left|D_{0}\right|=K\right)$, $P$ as the list of $K$ link-disjoint paths from each DC in $D_{0}$ to the requesting node, $b^{\prime}$ as the total offered bandwidth for the request, and $t^{\prime}$ as request departure time (s). Since the total offered bandwidth, $b^{\prime}$, is equally allocated to $K$ link-disjoint paths, the bandwidth on each path is $b_{p}^{\prime}=b^{\prime} / K$.

Algorithm 1 starts by constructing the auxiliary graph with the dummy node and dummy links, $G_{t}^{d}\left(V_{t}^{d}, E_{t}^{d}\right)$ (line 1). In find_paths, we first set the capacity of each link in $E_{t}^{d}$ to one bandwidth unit and use the Ford-Fulkerson's algorithm to find $K$ as the maximum number of link-disjoint paths from the dummy node to requesting node [21], [22]. find_paths also returns the flow graph, $G_{t}^{K}\left(V_{t}^{K}, E_{t}^{K}\right)$, where $V_{t}^{K}$ and $E_{t}^{K}$ are the actual nodes and links carrying the flow from the dummy node to the requesting node. If the maximum number of link-disjoint paths is larger than one (i.e., there are enough

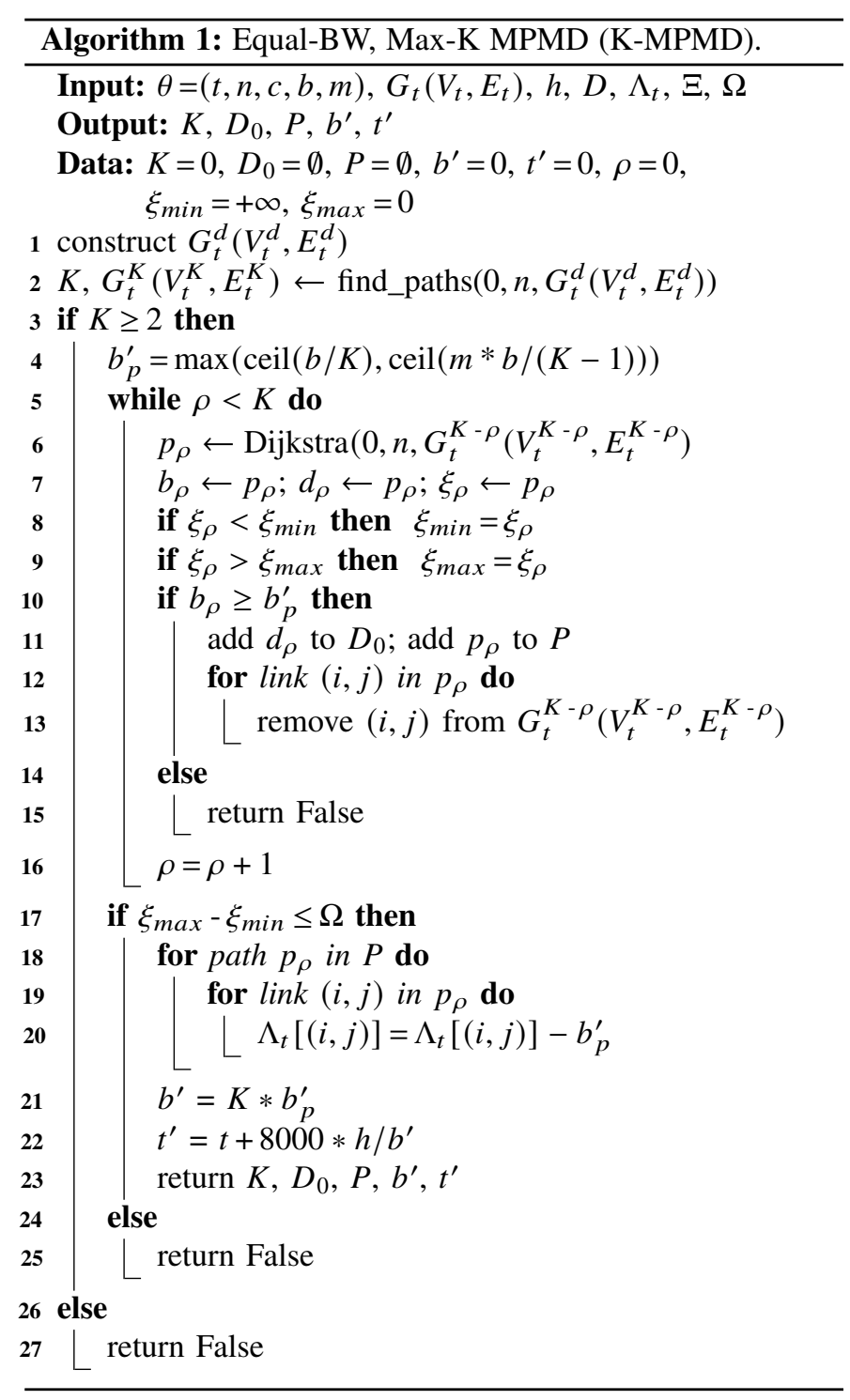

paths for multipath routing), the algorithm continues to find the minimum offered bandwidth on each path (i.e., $b_{p}^{\prime}$, which is rounded up to the nearest integer). The algorithm computes the minimum offered bandwidth on each path $\left(b_{p}^{\prime}\right)$ such that the constraints on the total requested bandwidth $(b)$ and degraded service $(m * b)$ in case a path is disrupted (i.e., there remains $K-1$ survivable paths) are fulfilled (line 4).

From lines 5 to 16, Algorithm 1 performs a path decomposition loop to find the actual link-disjoint paths from the dummy node to the requesting node, and equally allocate bandwidth to each path. Here, we use an augmented Dijkstra's algorithm to find the shortest path $\left(p_{\rho}\right)$ from the dummy node to the requesting node on the flow graph (line 6) [23]. In addition to the actual path, the algorithm also finds the available bandwidth of the path (i.e., $b_{\rho}$, bottleneck bandwidth), optimal DC (i.e., $d_{\rho}$, from which the desired content is retrieved), and total propagation delay of the path (i.e., $\xi_{\rho}$ ). Lines 8 and 9 update the propagation delay of the shortest path $\left(\xi_{\min }\right)$ and longest path $\left(\xi_{\max }\right)$ in each iteration. From lines 10 to 13 , the algorithm verifies that the available 
bandwidth of the path is enough for the requested bandwidth $\left(b_{\rho} \geq b_{p}^{\prime}\right)$ then adds optimal DC $d_{\rho}$ to set $D_{0}$, appends path $p_{\rho}$ to list $P$, and removes the links along path $p_{\rho}$ from $G_{t}^{K}\left(V_{t}^{K}, E_{t}^{K}\right)$. Note that, a path traced out from the dummy node on the flow graph always terminates at the requesting node since $G_{t}^{K}\left(V_{t}^{K}, E_{t}^{K}\right)$ is an acyclic, directed graph, and the path decomposition loop (lines 5-16) assuredly finds $K$ link-disjoint paths [21], [22]. However, the path decomposition loop provides no backtracking, thus it offers no guarantee that the total propagation delay of all paths is minimal. In case the available bandwidth of the path is not enough for the request, the content request is not admitted and the algorithm terminates with no result (line 15).

Once the bandwidth on each path is sufficient for the request, from lines 17 to 23 , the algorithm verifies that the differential propagation delay of the longest path and the shortest path fulfills DDC (i.e., $\xi_{\max }-\xi_{\min } \leq \Omega$ ); then it subtracts resources (i.e., capacity used on each link) from the network and returns $K, D_{0}, P, b^{\prime}$, and $t^{\prime}$. Lastly, if DDC is not fulfilled (line 26) or the number of paths from the dummy node to the requesting node is not enough for multipath routing (i.e., $K<2$, line 28), the content request is not admitted and the algorithm terminates with no result.

\section{Flexible MPMD (F-MPMD)}

K-MPMD exploits great path diversity by inversely multiplexing a content request over the maximum number of linkdisjoint paths, hence, it can reduce the total offered bandwidth. However, it may have potential shortcomings, e.g., K-MPMD rejects a content request if the available bandwidth on a path is not enough for the requested bandwidth. In another scenario, K-MPMD also rejects a content request if the propagation delay of a path does not fulfill DDC. This approach may not be optimal since the remaining paths (i.e., the paths whose bandwidth and propagation delay do not violate the two above-mentioned constraints) can still be used to admit the content request. In case a fewer number of paths is used, more bandwidth needs to be allocated to each path. Furthermore, if there exists only one path from the dummy node to the requesting node, the algorithm can still make its best effort to admit the request but provides no guarantee of survivability (i.e., no multipath routing). So, we derived an alternative approach, called flexible heuristic for the MPMD problem (FMPMD) as follows ( $\mathrm{F}$ for flexible).

As shown in Algorithm 2, F-MPMD shares the inputs, outputs, and most steps with K-MPMD. However, F-MPMD also provides $S$ as the output to indicate whether or not a service provisioning is survivable (i.e., survivable, $S=$ True). In contrast to Algorithm 1 (where the algorithm terminates if the number of paths is not enough for multipath provisioning, i.e, $K<2$ ), Algorithm 2 continues even if there is only one path from the dummy node to the requesting node (line 3 ). The code block between lines 4 and 9 computes the actual path(s) between the two nodes.

If there exists only one path from the dummy node to the requesting node and bandwidth on this path is enough for the requested bandwidth $\left(b_{0} \geq b\right)$, the content request is

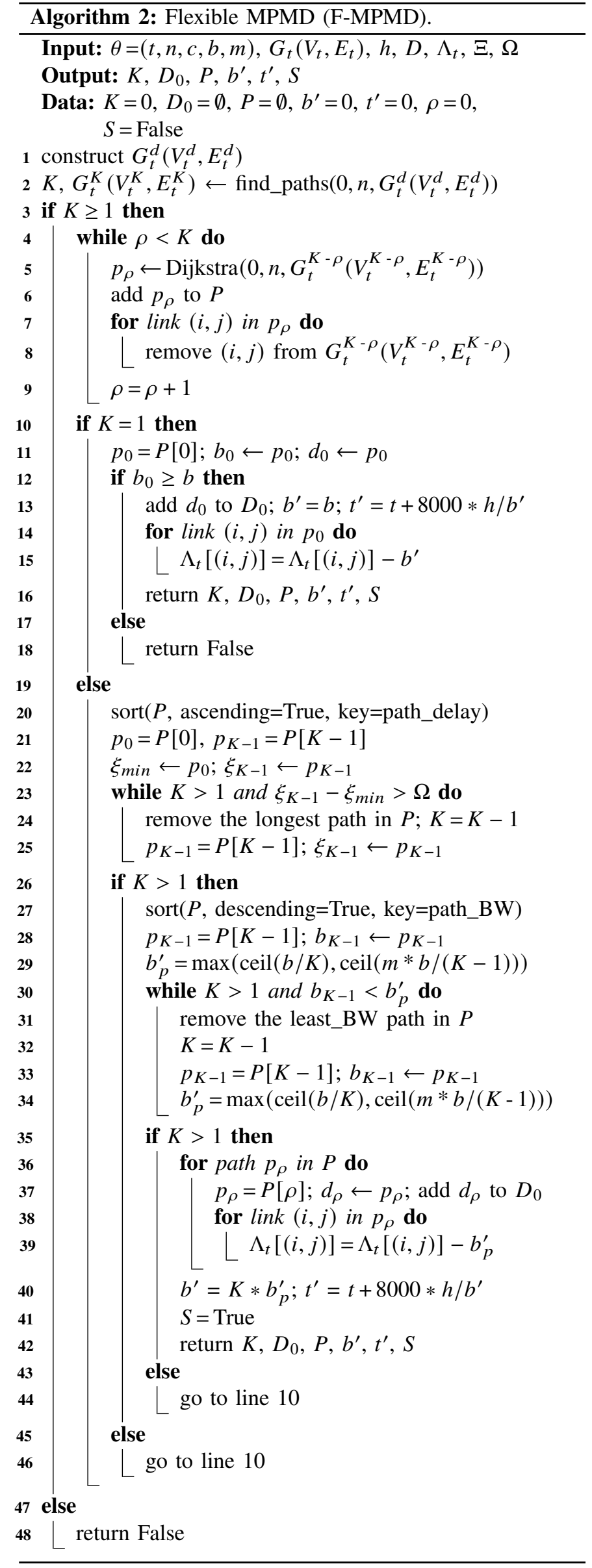


admitted, but provisioning is not survivable $(S=$ False, default value). Algorithm 2 updates the network and returns results (lines 10-16). In case the bandwidth on this single path from the dummy node to the requesting nodes is not enough for the requested bandwidth $\left(b_{0}<b\right)$, the content request is not admitted and the algorithm terminates with no result (line 18).

In case there are two or more link-disjoint paths from the dummy node to the requesting node (line 19), Algorithm 2 sorts the paths in $P$ by their total propagation delay in ascending order, sets the shortest path as the reference, and removes the paths whose propagation delay violates DDC (lines 20-25). In case the number of paths is more than one after removing the paths whose propagation delay violates DDC (line 26), Algorithm 2 continues to sort the paths in $P$ by their available bandwidths in descending order and removes the paths whose bandwidths are not enough to fulfill the requested bandwidth (lines 27-34). If the number of paths in $P$ is enough for multipath provisioning after both operations (i.e., removals of paths whose propagation delay and bandwidth do not fulfill the two-mentioned constraints, line 35), the content request is survivably provisioned (i.e., $S=$ True). Algorithm 2 subtracts resources (i.e., capacity used on each link) from the network and returns $K, D_{0}, P, b^{\prime}, t^{\prime}$, and $S$ (lines 36-42). In each step, if the number of paths remains one, Algorithm 2 returns to the scenario where there exists only one path from the dummy node to the requesting node (i.e., returns to line 10 , such as on lines 44 and 46).

In summary, Algorithm 2 is flexible in every step to provision a content request. The algorithm keeps removing invalid paths (i.e., paths whose propagation delay and bandwidth are insufficient for the request) until it can find the optimal $K$ to provision a content request.

The heuristic formulations for F-MPSD (i.e., flexible, MPSD) and DPP can be directly obtained from the F-MPMD formulations if, instead of the dummy node, the closest DC to the requesting node is used in Algorithm 2.

\section{Complexity Analysis}

In Algorithm 1, since the number of link-disjoint paths (i.e., $K$ ) is deterministic (e.g., number of link-disjoint paths must not exceed the nodal degree of the requesting node), the number of iterations inside the while loop (lines 5-16) is also deterministic. Moreover, as the number of links on each path is deterministic, the number of iterations inside the for loops (lines 12-13 and 19-22) is also deterministic. Note that the construction of a graph from another graph requires linear time complexity (i.e., $O\left(\left|V_{t}\right|+\left|E_{t}\right|\right)$ ), which is a non-dominant term. Algorithm 1's time complexity heavily depends on the time complexity of the method to find the maximum flow (line 2) and the method to find the shortest path (line 6) from the dummy node to the requesting node. In this study, we use the Ford-Fulkerson's algorithm to find the maximum flow from the dummy node to the requesting node whose time complexity is $O\left(\left|E_{t}^{d}\right|\right)$ (line 2). Moreover, we use the augmented Dijkstra's algorithm and a min-heap data structure to find the shortest path from the dummy node to the requesting node whose time complexity is $O\left(\left(\left|V_{t}^{K}\right|+\left|E_{t}^{K}\right|\right) * \log \left(\left|V_{t}^{K}\right|\right)\right)$. If we omit non- dominant terms and deterministic constants, the time complexity of Algorithm 1 is $O\left(\left|E_{t}^{d}\right|+\left(\left|V_{t}^{K}\right|+\left|E_{t}^{K}\right|\right) * \log \left(\left|V_{t}^{K}\right|\right)\right)$. Similarly, the time complexity of Algorithm 2 is $O\left(\left|E_{t}^{d}\right|+\left(\left|V_{t}^{K}\right|+\left|E_{t}^{K}\right|\right) * \log \left(\left|V_{t}^{K}\right|\right)\right)$ if we omit deterministic constants and non-dominant terms such as the sorting of the paths in $P$ (lines 20, 27).

\section{E. Service Probability}

In the previous subsections, we designed the algorithms for the MPMD problem against the dominant failure scenario, namely a single-link failure. The algorithms guarantee degraded service against a random single-link failure in an optical metro network. In this subsection, we compare the reliability of F-MPMD, F-MPSD, and DPP from a service probability perspective. We consider five typical scenarios, including a single-link failure $\left(L_{1}\right)$, a double-link failure $\left(L_{2}\right)$, a single-DC failure $\left(D_{1}\right)$, a double-DC failure $\left(D_{2}\right)$, a singlelink plus a single-DC failure $\left(L_{1}+D_{1}\right)$, and address the fundamental question: if a content request is already survivably provisioned (i.e., $K \geq 2$ ) and any of the failure scenarios occurs, what is the probability of fulfilling the requested degraded service? We define service probability, or $\Pi$, as the probability that the requested degraded service is guaranteed against a specific failure scenario. To simplify calculations without loss of generality, in this subsection, we assume that a link or a DC in an optical metro network is failed with equal probability.

Since F-MPMD, F-MPSD, and DPP are designed to guarantee degraded service against a single-link failure, their service probabilities in this scenario are $100 \%$, i.e., $\Pi_{\mathrm{F}-\mathrm{MPMD}}^{L_{1}}=\Pi_{\mathrm{F}-\mathrm{MPSD}}^{L_{1}}=\Pi_{\mathrm{DPP}}^{L_{1}}=100 \%$. Moreover, in contrast to F-MPSD and DPP, F-MPMD also provides protection against a single-DC failure, hence, $\Pi_{\mathrm{F}-\mathrm{MPMD}}^{D_{1}}=100 \%$. For other failure scenarios, we use $E_{\theta}$ to denote the set of physical links used by the content request $\theta, E_{\theta}^{\rho}$ to denote the sets of physical link(s) on each path, $\rho=\{0 . . K-1\}$, and derive the formulas for service probability as follows.

$$
\begin{gathered}
\Pi_{\mathrm{F}-\mathrm{MPSD}}^{D_{1}}=\Pi_{\mathrm{DPP}}^{D_{1}}=1-\frac{1}{|D|} . \\
\Pi_{\mathrm{F}-\mathrm{MPMD}}^{L_{2}}=\Pi_{\mathrm{F}-\mathrm{MPSD}}^{L_{2}}=1-\frac{\left(\begin{array}{c}
\left|E_{\theta}\right| \\
2
\end{array}\right)-\sum_{\rho=0:\left|E_{\theta}^{\rho}\right| \geq 2}^{K-1}\left(\begin{array}{c}
\left|E_{\theta}^{\rho}\right| \\
2
\end{array}\right)}{\left(\begin{array}{c}
\left|E_{t}\right| \\
2
\end{array}\right)} . \\
\Pi_{\mathrm{DPP}}^{L_{2}}=1-\frac{\left|E_{\theta}^{0}\right| *\left|E_{\theta}^{1}\right|}{\left(\begin{array}{c}
\left|E_{t}\right| \\
2
\end{array}\right)} . \\
\Pi_{\mathrm{F}-\mathrm{MPMD}}^{D_{2}}=1-\frac{\left(\begin{array}{c}
D_{0} \mid \\
2
\end{array}\right)}{\left(\begin{array}{c}
|D| \\
2
\end{array}\right)} . \\
\Pi_{\mathrm{F}-\mathrm{MPSD}}^{D_{2}}=\Pi_{\mathrm{DPP}}^{D_{2}}=1-\frac{|D|-1}{\left(\begin{array}{c}
|D| \\
2
\end{array}\right)} . \\
\Pi_{\mathrm{F}-\mathrm{MPMD}}^{L_{1}+D_{1}}=1-\frac{\left(\left|D_{0}\right|-1\right) *\left|E_{\theta}\right|}{|D| *\left|E_{t}\right|} . \\
\Pi_{\mathrm{F}-\mathrm{MPSD}}^{L_{1}+D_{1}}=\Pi_{\mathrm{DPP}}^{L_{1}+D_{1}}=1-\frac{\left|E_{\theta}\right|}{|D| *\left|E_{t}\right|} .
\end{gathered}
$$




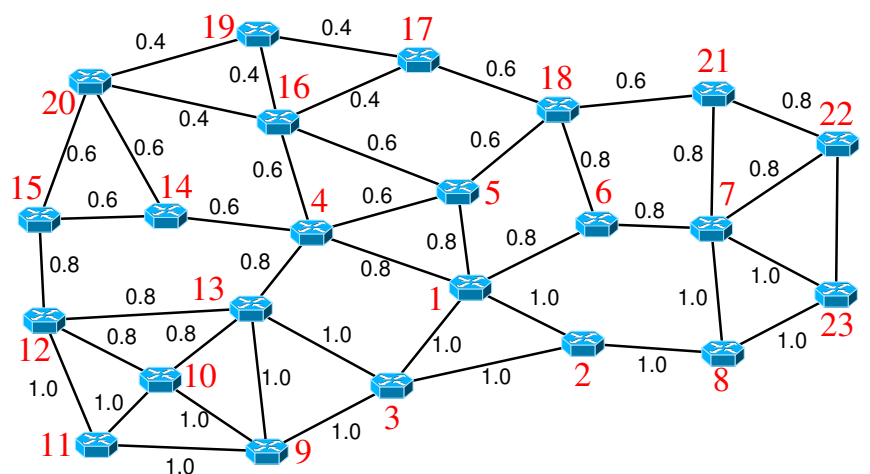

Fig. 3: Tokyo23 metro network with 23 nodes and 43 bidirectional links, where the number attached to each link, $\varphi^{i, j}$, is the normalized risk factor, $0 \leq \varphi^{i, j} \leq 1.0, \forall(i, j) \in E_{t}$. (Note: nodes closer to Tokyo Bay, in the Southeast, are at higher risk.)

Here, for example, for $D_{2}$ (i.e., a double-DC failure), we compute the number of combinations (without competition) which disrupt the requested degraded service (e.g., the numerator of the second term in (14) which is $\left({ }^{\left|D_{0}\right|} \begin{array}{c}2 \\ 2\end{array}\right)$ where $D_{0}$ is the set of serving DCs), the total number of combinations (e.g., the denominator of the second term in (14) which is $\left(\begin{array}{c}|D| \\ 2\end{array}\right)$ where $D$ is the set of content-hosting DCs), and the service probability against this failure scenario, $\Pi_{\mathrm{F}-\mathrm{MPMD}}^{D_{2}}=1-\left(\begin{array}{c}\left|D_{0}\right| \\ 2\end{array}\right) /\left(\begin{array}{c}|D| \\ 2\end{array}\right)$. In equations from (11) to (17), we derive the formulas to compute the service probability for each failure scenario and each protection scheme. We will use the above equations to show that, among the three schemes, F-MPMD has better service probability in most failure scenarios.

\section{ILlustrative Numerical Results}

\section{A. Physical Network and Simulation Setup}

1) Physical Network: To evaluate our proposed solutions, we use the Tokyo23 metro network covering an urban area up to tens of kilometers in diameter [24]. The Tokyo23 metro network has been designed using regional characteristics such as population distribution, locations of local government offices, and railway lines with the number of passengers getting on/off each station. It consists of 43 bidirectional links (i.e., 86 100-Gbps, unidirectional links) and 23 nodes, with each node located at each ward office building in the Tokyo metropolitan area. For risk modeling, we use information publicly available from other fields such as climatology, geology, environmental science, and construction engineering to estimate the risk of physical links. For the Tokyo23 metro network, we consider natural disasters such as earthquakes and tsunamis as the major cause of fiber cuts. A strong earthquake occurring in the sea (e.g., along Sagami Trough) may trigger tsunamis that ripple towards the Tokyo bay [25], [26]. Reasonably, we use a normalized risk factor to characterize the risk of each physical link in the Tokyo 23 metro network and assume that the links along the Tokyo bay coast are at higher risk as shown in Fig. 3.

2) Experiment Setup: To find the optimal experiment setup, we use the framework in Fig. 4 to simulate the network with $105000,205000,305000,405000$, and 505000 content requests whose arrivals follow a discrete Poisson process.

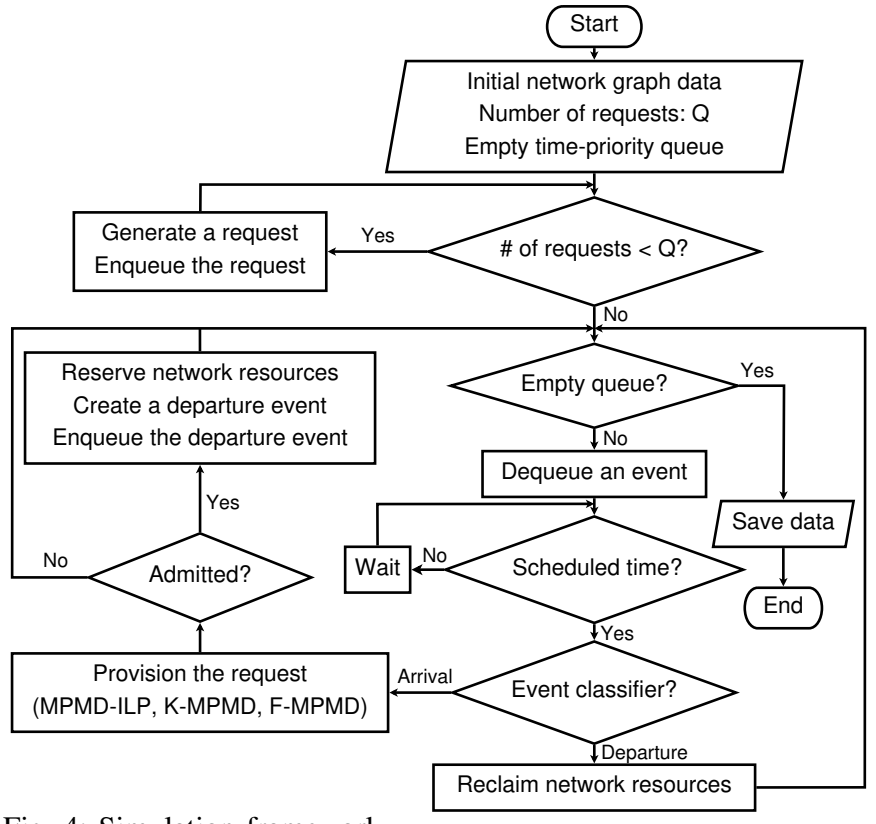

Fig. 4: Simulation framework.

We first generate all content requests and enqueue them in a time-priority queue. During simulation, each content request is dequeued from the queue and provisioned using one of the proposed schemes (MPMD-ILP, K-MPMD, F-MPMD). If a content request is admitted, network resources are reserved for it. These network resources will be reclaimed when the content's service ends. We found that the network requires approx. 5000 content requests to reach a steady state, and numerical results obtained for simulating 105000 and 505000 content requests are comparable. To reduce experiment time, below we report the numerical results for simulating 105000 content requests (i.e., first 5000 content requests are discarded).

In a dynamic network environment, the acceptance rate of incoming requests as a function of the arrival rates is crucial. In an ideal network with abundant resources (e.g., highcapacity links, super-fast computing capability in DCs/nodes, and contents replicated in multiple locations), the acceptance rate, or $\eta$, is $100 \%$. However, in practice, network resources are scarce, and when content requests arrive at a very high rate, the network becomes congested, and several incoming requests may be dropped (i.e., $\eta<100 \%$ ). In this study, we define the congestion point in a dynamic network as the arrival rate at which the network starts to drop several incoming requests, and use $\eta_{0}$ to denote this value. To run a network harder, with the same input setting, a service-provisioning scheme with a higher $\eta_{0}$ is desirable. In a congested network, a serviceprovisioning scheme with a higher $\eta$ is preferred since it can admit more requests. In the next sections, we will use $\eta_{0}$ and $\eta$ to evaluate our proposed service-provisioning schemes (MPMD-ILP, K-MPMD, and F-MPMD), and compare their performance to those of reference schemes (F-MPSD, DPP). Also, in Sections V.B, V.C, and V.D.1, we consider content requests which are survivably provisioned (i.e., $K \geq 2$ ). 


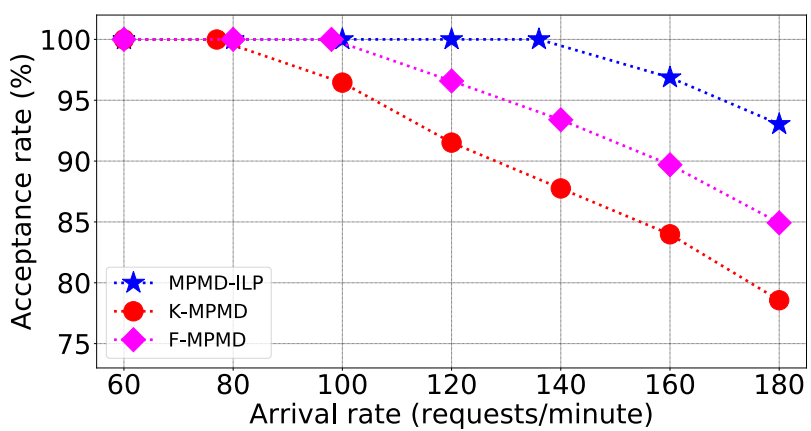

Fig. 5: Acceptance rate of MPMD-ILP, K-MPMD, and F-MPMD as a function of request arrival rates.

\section{B. MPMD-ILP vs. K-MPMD vs. F-MPMD}

Let us first compare the performance of MPMD-ILP, KMPMD, and F-MPMD. We consider a service provider with a content catalog of 10000 contents, whose size, $h$, ranges from 5 GB (e.g., a medium HD video) to $1000 \mathrm{~GB}$ (e.g., a long VR/AR video). We assume that, for each content request, the desired content is replicated in multiple DCs, i.e., $D=\{1,7,13,16\}$. Note that contents in DCs are dynamic, stateful, and require frequent updates (e.g., a content is replicated and synchronized in an edge DC by its popularity following a Zipf distribution [2]). However, the synchronization of contents among DCs is not considered in this study. The requested bandwidth, $b$, is uniformly selected from discrete values, ranging from $200 \mathrm{Mbps}$ (e.g., a stream for a VR head-mounted display worn by the user) to 2000 Mbps (e.g., an uncompressed VR/AR flow). The required level of degraded service for each request, $m$, is randomly selected from discrete values, $m \in\{0.5,0.7,1.0\}$, where $m=1.0$ denotes full protection in case a path is disrupted. The requesting node, $n$, is selected from the nodes not hosting the desired content following the population distribution. For DDC, we consider the propagation delay on fibers as the major delay in an optical network whose practical value is set to $2 \mathrm{~ms}$ (e.g., 6DoF VR immersive experience use case) [2], [8], [17].

In Fig. 5, we report the acceptance rate of MPMD-ILP, KMPMD, and F-MPMD at different request arrival rates. As expected, MPMD-ILP outperforms K-MPMD and F-MPMD. In fact, while K-MPMD and F-MPMD start dropping incoming requests around the arrival rates of 77 and 98 requests/minute, respectively, MPMD-ILP can run the same network harder and starts dropping incoming requests around the arrival rate of 136 requests/minute. In a congested network (e.g., where arrival rate is larger than 136 requests/minute), compared to K-MPMD and F-MPMD, respectively, MPMD-ILP approximately accepts $5 \%$ and $12 \%$ more incoming requests. Even though MPMD-ILP outperforms K-MPMD and F-MPMD, in a dynamic environment, F-MPMD, which outperforms KMPMD, is more desirable since it can find a fast solution.

In Table I, we report other relevant data for MPMD-ILP, KMPMD, and F-MPMD. On average, the number of paths per request (i.e., $K_{\text {avg }}$ ) decreases when moving from K-MPMD ( $\sim 3.5$ paths/request), to F-MPMD ( $\sim 3.3$ paths/request), and to MPMD-ILP ( $\sim 2.6$ paths/request) because K-MPMD always
TABLE I: MPMD-ILP vs. K-MPMD vs. F-MPMD

\begin{tabular}{|c|c|c|c|c|c|}
\hline$R$ & Schemes & $K_{\text {avg }}$ & $b_{a v g}^{\prime}$ & $b_{\text {avg }}^{\prime \prime}$ & $\xi$ Gavg \\
\hline \multirow{3}{*}{60} & MPMD-ILP & 2.6 & 1319.8 & 2432.6 & 45.5 \\
\hline & K-MPMD & 3.5 & 1272.1 & 2896.7 & 57.0 \\
\hline & F-MPMD & 3.5 & 1269.6 & 2887.4 & 56.9 \\
\hline \multirow{3}{*}{80} & MPMD-ILP & 2.6 & 1316.7 & 2433.2 & 45.7 \\
\hline & K-MPMD & 3.5 & 1268.4 & 2899.1 & 57.2 \\
\hline & F-MPMD & 3.5 & 1269.2 & 2891.2 & 57.0 \\
\hline \multirow{3}{*}{100} & MPMD-ILP & 2.6 & 1319.9 & 2451.9 & 45.7 \\
\hline & K-MPMD & 3.5 & 1258.3 & 2837.3 & 56.6 \\
\hline & F-MPMD & 3.4 & 1275.7 & 2891.9 & 56.9 \\
\hline \multirow{3}{*}{120} & MPMD-ILP & 2.6 & 1310.0 & 2473.5 & 46.4 \\
\hline & K-MPMD & 3.5 & 1230.1 & 2722.7 & 56.1 \\
\hline & F-MPMD & 3.3 & 1277.9 & 2842.6 & 56.4 \\
\hline \multirow{3}{*}{140} & MPMD-ILP & 2.6 & 1317.1 & 2530.4 & 47.0 \\
\hline & K-MPMD & 3.5 & 1219.5 & 2662.0 & 55.6 \\
\hline & F-MPMD & 3.2 & 1272.5 & 2766.5 & 55.7 \\
\hline \multirow{3}{*}{160} & MPMD-ILP & 2.6 & 1321.6 & 2572.4 & 48.0 \\
\hline & K-MPMD & 3.5 & 1200.4 & 2606.3 & 55.0 \\
\hline & F-MPMD & 3.2 & 1265.5 & 2697.1 & 55.1 \\
\hline \multirow{3}{*}{180} & MPMD-ILP & 2.6 & 1315.7 & 2507.4 & 49.1 \\
\hline & K-MPMD & 3.5 & 1172.4 & 2674.9 & 55.1 \\
\hline & F-MPMD & 3.1 & 1251.0 & 2628.9 & 54.5 \\
\hline
\end{tabular}

Data points in Table I are obtained by averaging over all accepted requests. Here, $R, K_{a v g}, b_{a v g}^{\prime}, b_{a v g}^{\prime \prime}$, and $\xi_{a v g}$ are request arrival rate (requests/minute), average number of paths per request (paths/request), average offered bandwidth (Mbps/request), average network bandwidth (Mbps/request), and average path propagation delay ( $\mu \mathrm{s} /$ path), respectively.

finds the maximum number of paths from the dummy node to the requesting node and equally allocates requested bandwidth to each path. On the contrary, MPMD-ILP finds just enough number of paths and allocates more bandwidth to each path to fulfill a request. Also, since F-MPMD first finds the maximum number of paths from the dummy node to the requesting node and then drops invalid paths (i.e., whose bandwidths or propagation delay is insufficient for the request), the average number of paths per request for F-MPMD is less than the average number of paths per request for K-MPMD.

Another notable observation is that, since the path decomposition loops in Algorithms 1 and 2 provide no guarantee to find the paths from each DC in $D_{0}$ to the requesting node whose total propagation delay is minimal, MPMD-ILP's average path propagation delay is less than K-MPMD and F-MPMD's average path propagation delay. On average, the path propagation delay (i.e., $\xi_{a v g}$ ) of MPMD-ILP is about $10 \mu \mathrm{s}$ less than the path propagation delay of K-MPMD and F-MPMD. As a result, even though MPMD-ILP offers more bandwidth per request (i.e., $b_{\text {avg }}^{\prime}$, on average, $11.5 \%$ more bandwidth), it uses less network bandwidth (i.e., $b_{a v g}^{\prime \prime}$ ). On average per request, compared to K-MPMD and F-MPMD, MPMD-ILP uses about $17.4 \%$ less network bandwidth.

\section{F-MPMD vs. F-MPSD vs. DPP}

In this subsection, we compare MPMD (using F-MPMD) with two reference protection schemes, namely F-MPSD and DPP. We use the same simulation setting as in Section V.B., and since the congestion point of DPP is lower than the congestion point of F-MPMD and F-MPSD, we also report numerical data for the arrival rate as low as 40 requests/minute.

1) Acceptance Rate and Latency: In Fig. 6, we report the acceptance rate of F-MPMD, F-MPSD, and DPP at different request arrival rates. Numerical data show that F-MPMD outperforms F-MPSD and DPP as it can run the network 


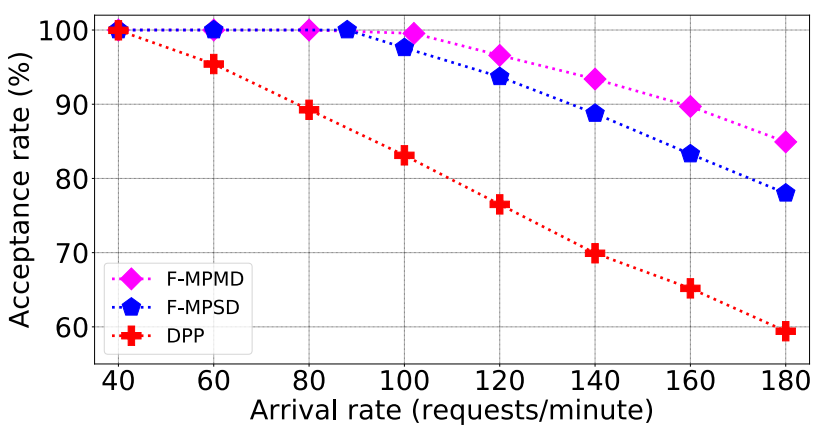

Fig. 6: Acceptance rates of F-MPMD, F-MPSD, and DPP as a function of request arrival rates.

TABLE II: F-MPMD vs. F-MPSD vs. DPP

\begin{tabular}{|c|c|c|c|c|c|}
\hline$R$ & Schemes & $K_{\text {avg }}$ & $b_{a v g}^{\prime}$ & $b_{a v g}^{\prime \prime}$ & $\xi_{a v g}$ \\
\hline \multirow{3}{*}{40} & F-MPMD & 3.5 & 1270.8 & 2893.2 & 57.0 \\
\cline { 2 - 6 } & F-MPSD & 3.5 & 1269.1 & 3831.3 & 75.2 \\
\cline { 2 - 6 } & DPP & 2.0 & 1895.0 & 3219.6 & 44.6 \\
\hline \multirow{3}{*}{60} & F-MPMD & 3.5 & 1269.6 & 2887.4 & 56.9 \\
\cline { 2 - 6 } & F-MPSD & 3.5 & 1274.1 & 3851.7 & 75.5 \\
\cline { 2 - 6 } & DPP & 2.0 & 1872.0 & 3176.1 & 44.5 \\
\hline \multirow{3}{*}{80} & F-MPMD & 3.4 & 1269.2 & 2891.2 & 57.0 \\
\cline { 2 - 6 } & F-MPSD & 3.4 & 1280.9 & 3854.6 & 75.2 \\
\cline { 2 - 6 } & DPP & 2.0 & 1853.0 & 3123.4 & 44.2 \\
\hline \multirow{3}{*}{100} & F-MPMD & 3.4 & 1275.7 & 2891.9 & 56.9 \\
\cline { 2 - 6 } & F-MPSD & 3.4 & 1268.3 & 3819.1 & 75.3 \\
\cline { 2 - 6 } & DPP & 2.0 & 1808.6 & 3043.8 & 44.3 \\
\hline \multirow{3}{*}{140} & F-MPMD & 3.3 & 1277.9 & 2842.6 & 56.4 \\
\cline { 2 - 6 } & F-MPSD & 3.3 & 1243.0 & 3729.8 & 75.1 \\
\cline { 2 - 6 } & DPP & 2.0 & 1763.1 & 2971.3 & 44.7 \\
\cline { 2 - 6 } & F-MPMD & 3.3 & 1272.5 & 2766.5 & 55.7 \\
\cline { 2 - 6 } & F-MPSD & 3.3 & 1219.7 & 3648.0 & 74.8 \\
\hline \multirow{3}{*}{160} & DPP & 2.0 & 1716.7 & 2899.6 & 45.1 \\
\cline { 2 - 6 } & F-MPMD & 3.3 & 1265.5 & 2697.1 & 55.1 \\
\cline { 2 - 6 } & F-MPSD & 3.3 & 1210.9 & 3598.4 & 74.7 \\
\hline \multirow{3}{*}{180} & DPP & 2.0 & 1683.6 & 2839.7 & 45.1 \\
\cline { 2 - 6 } & F-MPMD & 3.2 & 1251.0 & 2628.9 & 54.5 \\
\cline { 2 - 6 } & F-MPSD & 3.2 & 1169.4 & 3448.3 & 74.3 \\
\hline
\end{tabular}

Data points in Table II are obtained by averaging over all accepted requests. Here, $R, K_{a v g}, b_{a v g}^{\prime}, b_{a v g}^{\prime \prime}$, and $\xi_{a v g}$ are request arrival rate (requests/minute), average number of paths (paths/request), average offered bandwidth (Mbps/request), average network bandwidth (Mbps/request), and average path propagation delay ( $\mu \mathrm{s} / \mathrm{path})$, respectively.

harder with a higher congestion point. In detail, the congestion points of F-MPMD, F-MPSD, and DPP are 102, 88, and 40 requests/minute, respectively. In a congested network, compared to F-MPSD and DPP, respectively, F-MPMD accepts approximately $4 \%$ and $15 \%$ more incoming requests.

In Table II, we report the relevant data for F-MPMD, FMPSD, and DPP. Since both F-MPMD and F-MPSD rely on multipath routing, they use significantly less bandwidth. Compared to DPP, on average, F-MPMD uses about $39.5 \%$ less bandwidth per request (i.e., $b_{a v g}^{\prime}$ ). As another observation, among the three service-provisioning schemes, F-MPMD uses least network bandwidth per request (i.e., $b_{a v g}^{\prime \prime}$ ), and compared to F-MPSD, it can save up to $30 \%$. Moreover, on average, the path propagation delay of F-MPMD (i.e., $\xi_{a v g}$ ) is approximately $20 \mu \mathrm{s}$ less than the path propagation delay of F-MPSD, making F-MPMD more suitable for emerging services which have stringent latency constraint. Lastly, since DPP uses only two paths (i.e., the first and second shortest paths from the closest DC to the requesting node), the average propagation delay per path used by DPP is least among the three service-
TABLE III: Average Service Probability in Different Failure Scenarios.

\begin{tabular}{|c|c|c|c|}
\hline Scenario & F-MPMD & F-MPSD & DPP \\
\hline$L_{1}$ & $100 \%$ & $100 \%$ & $100 \%$ \\
\hline$D_{1}$ & $100 \%$ & $71.3 \%$ & $71.3 \%$ \\
\hline$L_{2}$ & $99.4 \%$ & $98.9 \%$ & $99.9 \%$ \\
\hline$D_{2}$ & $60.1 \%$ & $54.2 \%$ & $54.2 \%$ \\
\hline$L_{1}+D_{1}$ & $94.7 \%$ & $96.5 \%$ & $98.6 \%$ \\
\hline
\end{tabular}

Here, $L_{1}, D_{1}, L_{2}, D_{2}$, and $L_{1}+D_{1}$ are for a single-link, a single-DC, a double-link, a double-DC, and one link plus one DC failure scenarios, respectively.

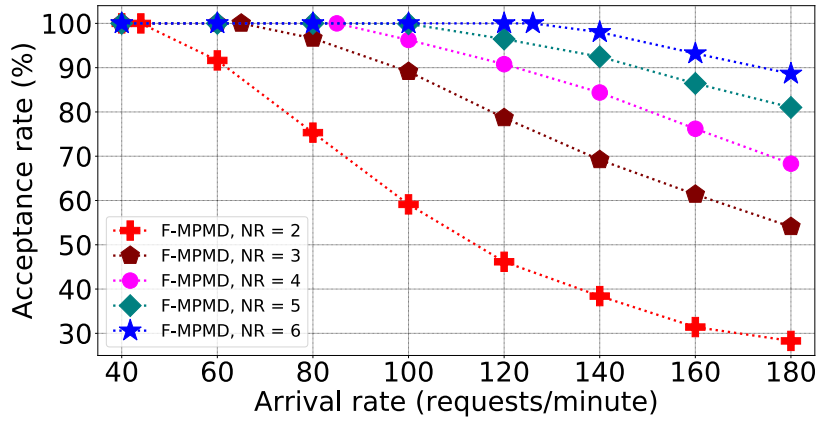

Fig. 7: Acceptance rate of F-MPMD as a function of number of content replicas (NR) at different request arrival rates.

provisioning schemes (F-MPMD, F-MPSD, and DPP).

2) Service Probability: We now compare the service probability of F-MPMD to the service probability of F-MPSD and DPP. Here, we set the request arrival rate to 40 requests/minute (i.e., $\eta=100 \%$ for F-MPMD, F-MPSD, and DPP as in Fig. 6), and compute the average service probability per request for each failure scenario using the formulas in Section IV.E. We report the results in Table III.

As expected, F-MPMD, F-MPSD, and DPP all guarantee degraded service against a random single-link failure on the physical network (i.e., $\Pi_{\mathrm{F}-\mathrm{MPMD}}^{L_{1}}=\Pi_{\mathrm{F}-\mathrm{MPSD}}^{L_{1}}=\Pi_{\mathrm{DPP}}^{L_{1}}=100 \%$ ). Moreover, since F-MPMD offers protection against a singleDC failure, its service probability is $100 \%$ in this scenario (i.e., $\Pi_{\mathrm{F}-\mathrm{MPMD}}^{D_{1}}=100 \%$ ) while F-MPSD and DPP provide no service guarantee (i.e., $\Pi_{\mathrm{F}-\mathrm{MPSD}}^{D_{1}}=\Pi_{\mathrm{DPP}}^{D_{1}}=71.3 \%$ ). We also see that, since DPP uses only two paths, for each request, the number physical links used by DPP is fewer than the number physical links used by F-MPMD and F-MPSD. In other words, the number the double-link failure combinations disrupting DPP is lower than the number the double-link failure combinations disrupting F-MPMD and F-MPSD. As a result, among the three protection schemes, DPP has the highest service probability against a double-link failure (i.e., $\Pi_{\mathrm{DPP}}^{L_{2}}=99.9 \%$ ). Similarly, compared to F-MPSD, F-MPMD tends to use shorter paths (refer to $\xi_{a v g}$ in Table II); hence, it has higher service probability against a double-link failure $\left(\Pi_{\mathrm{F}-\mathrm{MPMD}}^{L_{2}}=99.4 \%\right.$, compared to $\left.\Pi_{\mathrm{F}-\mathrm{MPSD}}^{L_{2}}=98.9 \%\right)$. In the scenario where there are two simultaneous failures in two distinct DCs, F-MPMD outperforms F-MPSD and DPP in terms of service probability $\left(\Pi_{\mathrm{F}-\mathrm{MPMD}}^{D_{2}}=60.1 \%\right.$ compared to $\left.\Pi_{\mathrm{F}-\mathrm{MPSD}}^{D_{2}}=\Pi_{\mathrm{DPP}}^{D_{2}}=54.2 \%\right)$ because F-MPMD uses multiple DCs while F-MPSD and DPP use only one DC for each request. For the last scenario where one link plus one DC fail simultaneously (i.e., $L_{1}+D_{1}$ ), with the same total number of links (i.e., $\left|E_{\theta}\right|$ in Eqns. (16) 


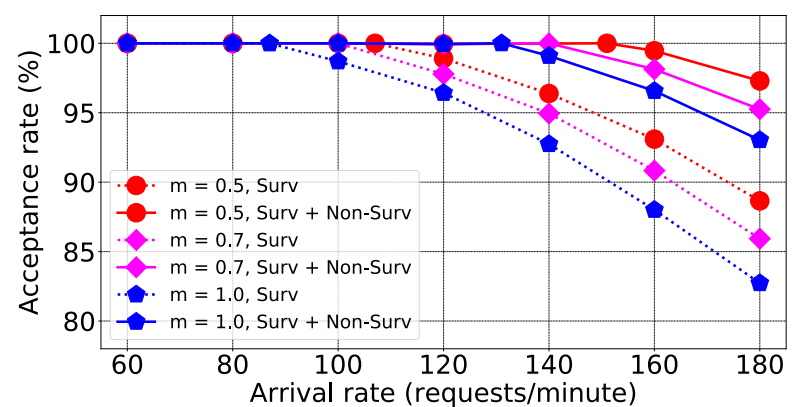

Fig. 8: Acceptance rate of F-MPMD as a function of request arrival rates at three different levels of degraded service.

and (17)), the number of combinations disrupting F-MPMD is $\left|D_{0}\right|-1$ times the number of combinations disrupting FMPSD and DPP. Hence, compared to F-MPSD and DPP, F-MPMD has lowest service probability $\left(\Pi_{\mathrm{F}-\mathrm{MPMD}}^{L_{1}+D_{1}}=94.7 \%\right.$, $\Pi_{\mathrm{F}-\mathrm{MPSD}}^{L_{1}+D_{1}}=96.5 \%$, and $\left.\Pi_{\mathrm{DPP}}^{L_{1}+D_{1}}=98.6 \%\right)$.

\section{F-MPMD}

1) Number of Content Replicas (NR): In this subsection, we use the same simulation setting as in Section V.C. and obtain numerical results for F-MPMD for increasing number of content replicas (NR) in the network from two to six (i.e., $N R=2, D=\{1,4\} ; N R=3, D=\{1,4,7\} ; N R=4$, $D=\{1,4,7,10\} ; N R=5, D=\{1,4,7,10,13\} ;$ and $N R=6$, $D=\{1,4,7,10,13,16\})$. As shown in Fig. 7, increasing the number of content replicas from $N R=2$ to $N R=3$ significantly improves the acceptance rate of incoming requests. In detail, the congestion point is increased from 44 to 65 requests/minute; and in a congested network, with $N R=3$, compared to $N R=2$, F-MPMD accepts around $25 \%$ more incoming requests, while acceptance rate improves more slowly when content replicas are further increased to 4,5 , and 6 . In general, adding more content replicas increases the acceptance rate, but it also implies more synchronization overhead. For this trade-off, only a content provider knows which option is cost-optimal. Therefore, the decision on the number of content replicas in a specific network may vary from content provider to content provider.

2) Survivability (Surv) vs. Non-Survivability (Non-Surv): To this end, we show how many more content requests F-MPMD can admit even though their survivability is not guaranteed. We reports results for three distinct levels of degraded service (i.e., $m=0.5,0.7$, and 1.0).

As shown in Fig. 8, the dotted lines denote acceptance rates where admitted requests are survivable in case a path is disrupted (i.e., $S=$ True in Algorithm 2) while the solid lines represent acceptance rates where admitted requests can be non-survivable. Considering the dotted lines, switching the levels of degraded service from 1.0 (i.e., full protection), down to 0.7 , and to 0.5 increases the congestion points from 87 , to 100, and to 107 (requests/minute), respectively. Furthermore, the solid lines show how many more content requests FMPMD can admit even though it provides no guarantee of survivability against a single-link failure. By admitting several requests without ensuring survivability (solid lines),
F-MPMD can run the same network much harder and only starts dropping incoming requests at the arrival rates of 131, 140 , and 152 (requests/minute), for $\mathrm{m}=0.5, \mathrm{~m}=0.7$, and $\mathrm{m}=$ 1.0 , respectively. In a highly congested network (e.g., arrival rate 180 (requests/minute)), compared to the scheme where B-MPMD ensures survivability against a single-link failure, B-MPMD can admit approx. 10\% more incoming requests.

\section{CONCLUSION}

We proposed a reliable service-provisioning scheme that inversely multiplexes a dynamic content request over multiple link-disjoint paths from multiple data centers using manycast. We developed an integer linear program and two scalable heuristics for the proposed scheme and used them to solve various complex network instances. Numerical data show that, compared to conventional service-provisioning schemes such as multipath routing from a single data center and dedicatedpath protection, our proposed service-provisioning scheme efficiently utilizes network resources, admits more requests, improves reliability, reduces latency; hence, it is very suitable for emerging content-based services.

\section{ACKNOWLEDGMENT}

This work is supported by National Science Foundation (NSF), Japan-US Network Opportunity 2 (JUNO2), Grant No. 1818972. Preliminary results of this work were presented at the Optical Fiber Communication (OFC) conference, June 2021.

\section{REFERENCES}

[1] L. Peterson, A. Al-Shabibi, T. Anshutz, S. Baker, A. Bavier, S. Das, J. Hart, G. Palukar, and W. Snow, "Central office re-architected as a data center," IEEE Communications Magazine, vol. 54, no. 10, pp. 96101, 2016.

[2] Metro-Haul, "Definition of use cases, service requirements and KPIs," Deliverable D2.1, 2018.

[3] C. Natalino, A. de Sousa, L. Wosinska, and M. Furdek, "Content placement in 5G-enabled edge/core data center networks resilient to link cut attacks," Networks, vol. 75, no. 4, pp. 392-404, 2020.

[4] Cisco, "Cisco visual networking index (VNI): Forecast and trends 2017 - 2022," White Paper, 2019.

[5] Qualcomm, "Augmented and virtual reality: The first wave of 5G killer apps," White Paper, 2017.

[6] W. Saad, M. Bennis, and M. Chen, "A vision of $6 \mathrm{G}$ wireless systems: Applications, trends, technologies, and open research problems," IEEE Network, vol. 34, no. 3, pp. 134-142, 2020.

[7] B. Mukherjee, Optical WDM Networks. Springer, 2006.

[8] W. Zhang, J. Tang, C. Wang, and S. de Soysa, "Reliable adaptive multipath provisioning with bandwidth and differential delay constraints," in Proc. of IEEE INFOCOM, 2010.

[9] C. S. K. Vadrevu, R. Wang, M. Tornatore, C. U. Martel, and B. Mukherjee, "Degraded service provisioning in mixed-line-rate WDM backbone networks using multipath routing," IEEE/ACM Transactions on Networking, vol. 22, no. 3, pp. 840-849, 2014.

[10] P. Gill, N. Jain, and N. Nagappan, "Understanding network failures in data centers: Measurement, analysis, and implications," in Proc. of ACM SIGCOMM, 2011.

[11] G. Le, A. Marotta, S. Ferdousi, S. Xu, Y. Hirota, Y. Awaji, M. Tornatore and B. Mukherjee, "Logical network mapping with content connectivity against multiple link failures in optical metro networks," in Proc. of IEEE ANTS, 2019.

[12] G. Le, S. Ferdousi, A. Marotta, S. Xu, Y. Hirota, Y. Awaji, M. Tornatore, and B. Mukherjee, "Survivable virtual network mapping with content connectivity against multiple link failures in optical metro networks," IEEE/OSA Journal of Optical Communications and Networking, vol. 12, no. 11 , pp. 301-311, 2020. 
[13] — - "Reliable provisioning for dynamic content requests in optical metro networks," in Proc. of IEEE/OSA OFC, 2021.

[14] J. Zhang, K. Zhu, and B. Mukherjee, "Backup reprovisioning to remedy the effect of multiple link failures in WDM mesh networks," IEEE Journal on Selected Areas in Communications, vol. 24, no. 8, pp. 57-67, 2006.

[15] L. Ruan and N. Xiao, "Survivable multipath routing and spectrum allocation in OFDM-based flexible optical networks," IEEE/OSA Journal of Optical Communications and Networking, vol. 5, no. 3, pp. 172-182, 2013.

[16] L. Ruan and Y. Zheng, "Dynamic survivable multipath routing and spectrum allocation in OFDM-based flexible optical networks," IEEE/OSA Journal of Optical Communications and Networking, vol. 6, no. 1, pp. 77-85, 2014.

[17] S. Huang, C. U. Martel, and B. Mukherjee, "Survivable multipath provisioning with differential delay constraint in telecom mesh networks," IEEE/ACM Transactions on Networking, vol. 19, no. 3, pp. 657-669, 2011.

[18] N. Charbonneau and V. M. Vokkarane, "Routing and wavelength assignment of static manycast demands over all-optical wavelength-routed WDM networks," IEEE/OSA Journal of Optical Communications and Networking, vol. 2, no. 7, pp. 442-455, 2010.

[19] X. Li, S. Yin, X. Wang, Y. Zhou, Y. Zhao, S. Huang, and J. Zhang, "Content placement with maximum number of end-to-content paths in $K$ node (edge) content connected optical datacenter networks," IEEE/OSA Journal of Optical Communications and Networking, vol. 9, no. 1, pp. 53-66, 2017.

[20] S. S. Savas, F. Dikbiyik, M. F. Habib, M. Tornatore, and B. Mukherjee, "Disaster-aware service provisioning with manycasting in cloud networks," Photonic Network Communications, vol. 28, no. 2, p. 123-134, 2014.

[21] L. R. Ford and D. R. Fulkerson, "Maximal flow through a network," Canadian Journal of Mathematics, vol. 8, pp. 399-404, 1956.

[22] J. Kleinberg and E. Tardos, Algorithm Design. Pearson Education Inc., 2006.

[23] E. W. Dijkstra, "A note on two problems in connexion with graphs," Numerische Mathematik, vol. 1, no. 1, pp. 269-271, 1959.

[24] T. Tachibana, Y. Hirota, K. Suzuki, T. Tsuritani, and H. Hasegawa, "Construction algorithm of metropolitan area network based on regional characteristics in Japan: A case of Tokyo metropolitan area," IEICE Technical Report, vol. 119, no. 221, 2019.

[25] The Headquarters for Earthquake Research Promotion, "National seismic hazard maps for Japan," Technical Report, 2020.

[26] R. Nagai, T. Takabatake, M. Esteban, H. Ishii, and T. Shibayama, "Tsunami risk hazard in Tokyo bay: The challenge of future sea level rise," International Journal of Disaster Risk Reduction, vol. 45, p. 101321, 2020. 